\title{
A class of completely monotonic functions involving the polygamma functions
}

\section{Li-Chun Liang ${ }^{1}$ (D) Li-Fei Zheng ${ }^{1 *}$ (D) and Aying Wan $^{2}$ (B)}

\author{
"Correspondence: \\ zhenglifei@nwafu.edu.cn \\ ${ }^{1}$ College of Science, Northwest A\&F \\ University, Yangling, Shaanxi, \\ 712100, P.R. China \\ Full list of author information is \\ available at the end of the article
}

\begin{abstract}
Let $\Gamma(x)$ denote the classical Euler gamma function. We set $\psi_{n}(x)=(-1)^{n-1} \psi^{(n)}(x)$ $(n \in \mathbb{N})$, where $\psi^{(n)}(x)$ denotes the $n$th derivative of the psi function $\psi(x)=\Gamma^{\prime}(x) / \Gamma(x)$. For $\lambda, \alpha, \beta \in \mathbb{R}$ and $m, n \in \mathbb{N}$, we establish necessary and sufficient conditions for the functions

$$
L(x ; \lambda, \alpha, \beta)=\psi_{m+n}(x)-\lambda \psi_{m}(x+\alpha) \psi_{n}(x+\beta)
$$

and $-L(x ; \lambda, \alpha, \beta)$ to be completely monotonic on $(-\min (\alpha, \beta, 0), \infty)$.

As a result, we generalize and refine some inequalities involving the polygamma functions and also give some inequalities in terms of the ratio of gamma functions.

MSC: Primary 33B15; secondary 26007

Keywords: Polygamma functions; Inequalities; Psi function; Complete monotonicity
\end{abstract}

\section{Introduction}

We know that Euler's gamma function is defined by $\Gamma(x)=\int_{0}^{\infty} t^{x-1} e^{-t} d t$ for $x>0$. The psi or digamma function is its logarithmic derivative

$$
\psi(x)=\frac{d}{d x} \ln \Gamma(x)=\frac{\Gamma^{\prime}(x)}{\Gamma(x)}
$$

whose derivatives $\psi^{\prime}(x)$ and $\psi^{\prime \prime}(x)$ are called the trigamma and tetragamma functions, respectively. The polygamma functions are higher-order derivative

$$
\psi^{(n)}(x)=(-1)^{n-1} \int_{0}^{\infty} e^{-x t} \frac{t^{n}}{1-e^{-t}} d t=(-1)^{n-1} n ! \sum_{k=0}^{\infty} \frac{1}{(x+k)^{n+1}},
$$

where $n \in \mathbb{N}$. The Gamma function's history and its development are given in [1].

After Euler discovered the gamma function, some scholars studied the fundamental properties of gamma, digamma, and polygamma functions, see [2-5]. These functions are important in the fields of engineering, physics, inequality theory, or statistics, and many inequalities involving these functions have been obtained through monotonicity or convexity properties, see [6-16].

(c) The Author(s) 2022. This article is licensed under a Creative Commons Attribution 4.0 International License, which permits use, sharing, adaptation, distribution and reproduction in any medium or format, as long as you give appropriate credit to the original author(s) and the source, provide a link to the Creative Commons licence, and indicate if changes were made. The images or other third party material in this article are included in the article's Creative Commons licence, unless indicated otherwise in a credit line to the material. If material is not included in the article's Creative Commons licence and your intended use is not permitted by statutory regulation or exceeds the permitted use, you will need to obtain permission directly from the copyright holder. To view a copy of this licence, visit http://creativecommons.org/licenses/by/4.0/. 
A function $f$ is said to be completely monotonic on an interval $I$ if $f$ has derivatives of all orders on $I$ and $(-1)^{n} f^{(n)}(x) \geq 0, x \in I, n \geq 0$ (see [17]). A function $f$ is said to be strictly completely monotonic if $(-1)^{n} f^{(n)}(x)>0$. The Bernstein-Widder Theorem [17, Theorem 12b, p. 161] states that $f$ is completely monotonic on $(0, \infty)$ if and only if

$$
f(x)=\int_{0}^{\infty} e^{-x t} d \alpha(t)
$$

where $\alpha(t)$ is nondecreasing such that the integral converges for $x>0$. Completely monotonic functions have attracted the attention of many researchers in various fields (see $[8,18-24])$.

The following asymptotic formulas are often encountered in many papers (see [5]).

$$
\begin{aligned}
& \psi^{(n)}(x+1)=\psi^{(n)}(x)+(-1)^{n} \frac{n !}{x^{n+1}}, \quad(x>0 ; n=0,1, \ldots), \\
& \ln \Gamma(x)=\left(x-\frac{1}{2}\right) \ln x-x+\frac{1}{2} \ln (2 \pi)+\frac{1}{12 x}+\cdots, \quad(x \rightarrow \infty), \\
& \psi(x) \sim \ln x-\frac{1}{2 x}-\frac{1}{12 x^{2}}+\frac{1}{120 x^{4}}-\cdots, \quad(x \rightarrow \infty), \\
& (-1)^{n-1} \psi^{n}(x) \sim\left(\frac{(n-1) !}{x^{n}}+\frac{n !}{2 x^{n+1}}+\frac{(n+1) !}{12 x^{n+2}}-\cdots\right), \quad(x \rightarrow \infty, n=1,2, \ldots) .
\end{aligned}
$$

For the sake of convenience, we set $\psi_{n}(x)=(-1)^{n-1} \psi^{(n)}(x)$ for $n \in \mathbb{N}$.

From (1.2) and the Bernstein-Widder Theorem, we know that $\psi_{n}(x)$ is strictly completely monotonic on $(0, \infty)$. From (1.3) and (1.6), we have

$$
\begin{aligned}
& \lim _{x \rightarrow 0^{+}} x^{n+1} \psi_{n}(x)=n !, \\
& \lim _{x \rightarrow \infty} x^{n} \psi_{n}(x)=(n-1) !
\end{aligned}
$$

which easily yields that $\lim _{x \rightarrow 0^{+}} \psi_{n}(x)=\infty$ and $\lim _{x \rightarrow \infty} \psi_{n}(x)=0$.

In order to prove [25, Theorem 4.8], Alzer provided

$$
\psi_{1}^{2}(x)-\psi_{2}(x)>0, \quad x>0,
$$

which was verified in a distinct way in [26, Lemma 1.1]. Furthermore, it is worthwhile to notice that [27, Lemma 1.2] is a generalization of the inequality (1.9) and is used to establish many interesting results (see [26, 27]). From [27, Theorem 2.2], it follows that

$$
\psi_{1}^{2}\left(x+\frac{1}{2}\right)-\psi_{2}(x)<0, \quad x>0 .
$$

In light of (1.9) and (1.10), a novel question was raised in [27], which asks whether it is possible that there exist constants $\alpha$ and $\beta$ such that

$$
\psi_{1}^{2}(x+\alpha)-\psi_{2}(x)>0
$$

and

$$
\psi_{1}^{2}(x+\beta)-\psi_{2}(x)<0, \quad x>0 .
$$


Recently, Qi and Guo showed in [28, Theorem 1] that for $\alpha \in \mathbb{R}$, the function

$$
f(x ; \alpha)=\psi_{1}^{2}(x+\alpha)-\psi_{2}(x)
$$

is completely monotonic on $(-\min (0, \alpha), \infty)$ if and only if $\alpha \leq 0$, and so is the function $-f(x ; \alpha)$ if

$$
\alpha \geq \sup _{x \in(0, \infty)} \frac{x}{\rho^{-1}\left(e^{2 x}\left(2(x+1)^{2}-1\right)\right)},
$$

where $\rho(x)=x \operatorname{coth} x$ for $x>0$ and $\rho^{-1}(x)$ is the inverse function of $\rho(x)$.

In addition, it was shown in [28, Theorem 3$]$ that the function

$$
f_{\lambda}(x)=\psi_{1}^{2}(x)-\lambda \psi_{2}(x)
$$

is completely monotonic on $(0, \infty)$ if and only if $\lambda \leq 1$.

Besides the preceding conclusions invoked, we can refer to more references on results extending (1.9) or (1.14) (see [19, 20, 24, 29-35]).

In view of (1.13), we define the function $L(x ; \lambda, \alpha, \beta)$ for $\lambda, \alpha, \beta \in \mathbb{R}, \eta=\min (\alpha, \beta, 0)$ and $m, n \in \mathbb{N}$ as follows:

$$
L(x ; \lambda, \alpha, \beta)=\psi_{m+n}(x)-\lambda \psi_{m}(x+\alpha) \psi_{n}(x+\beta)
$$

with respect to $x \in(-\eta, \infty)$.

Then it is a question to put forward: Do sufficient and necessary conditions exist such that $L(x ; \lambda, \alpha, \beta)$ is completely monotonic?

The aim of this paper is to solve this question and then apply it to obtain more inequalities involving ratios, differences of digamma and polygamma functions.

A detailed plan of this paper is as follows: In Sect. 2, we give detailed proof of our main results. In Sect. 3, some more inequalities for ratios of gamma functions are obtained with the aid of Theorem 3.1.

\section{A lemma}

In order to prove our main results, we need the following:

Lemma 1 For $\alpha, \beta \in \mathbb{R}$ and $t>0$, let the function $\phi(x)$ be defined on $(0,1)$ by

$$
\phi(x)=\frac{t\left(1-e^{-t}\right)}{1-e^{-t x}} \frac{x(1-x)}{1-e^{-t(1-x)}} e^{-\alpha t x} e^{-\beta t(1-x)} .
$$

Then the following statements are true:

(1) For $\beta-\alpha \geq \frac{1}{2}$, the function $\phi(x)$ is increasing from $(0,1)$ onto $\left(e^{-\beta t}, e^{-\alpha t}\right)$;

(2) For $\beta-\alpha \leq-\frac{1}{2}$, the function $\phi(x)$ is decreasing from $(0,1)$ onto $\left(e^{-\alpha t}, e^{-\beta t}\right)$;

(3) For $-1 / 2<\alpha-\beta<0$, there exists $t_{0} \geq 0$ such that when $0<t<t_{0}$, the function $\phi(x)$ is increasing from $(0,1)$ onto $\left(e^{-\beta t}, e^{-\alpha t}\right)$;

(4) For $0<\alpha-\beta<1 / 2$, there exists $t_{0} \geq 0$ such that when $0<t<t_{0}$, the function $\phi(x)$ is decreasing from $(0,1)$ onto $\left(e^{-\alpha t}, e^{-\beta t}\right)$; 
(5) For $|\alpha-\beta|<\frac{1}{2}$, there exists $t_{0} \geq 0$ such that when $t>t_{0}$, the function $\phi(x)$ has a unique maximum point $x_{0}(t)$ on $(0,1)$, that is, $\phi(x)$ is increasing on $\left(0, x_{0}(t)\right)$ and decreasing on $\left(x_{0}(t), 1\right)$. In particular, if $\alpha=\beta$, then $x_{0}(t)=1 / 2$.

Proof Differentiating $v(x)=\ln \phi(x)$ yields

$$
v^{\prime}(x)=t(\omega(t x)-\omega(t(1-x))+\beta-\alpha),
$$

where

$$
\omega(x)=\frac{1}{x}-\frac{1}{e^{x}-1}, \quad x>0 .
$$

It is not difficult to show that $\omega(x)$ is decreasing from $(0, \infty)$ onto $\left(0, \frac{1}{2}\right)$ by noting that

$$
\omega^{\prime}(x)=\frac{\left[\left(\frac{x}{2}\right)^{2}-\left(\sinh \left(\frac{x}{2}\right)\right)^{2}\right]}{\left(x \sinh \left(\frac{x}{2}\right)\right)^{2}}<0 .
$$

Apparently, we have

$$
v^{\prime \prime}(x)=t^{2}\left(\omega^{\prime}(t x)+\omega^{\prime}(t(1-x))\right)<0,
$$

so that

$$
v^{\prime}(1)=t(-(\omega(0)-\omega(t))+\beta-\alpha)<v^{\prime}(x)<t(\omega(0)-\omega(t)+\beta-\alpha)=v^{\prime}(0) .
$$

For $\beta-\alpha \geq \frac{1}{2}$, from $\omega^{\prime}(x)<0$ and $0<\omega(x)<1 / 2$, we see that $v^{\prime}(1)>0$, that is, $v(x)$ is increasing on $(0,1)$, which immediately yields

$$
e^{-\beta t}<\phi(x)<e^{-\alpha t} .
$$

For $\beta-\alpha \leq-\frac{1}{2}$, a similar argument yields $v^{\prime}(0)<0$, and therefore $v(x)$ is decreasing on $(0,1)$, which leads to the inversed inequality of $(2.5)$.

If $0<|\alpha-\beta|<\frac{1}{2}$, then there exists $t_{0}>0$ satisfying $\omega(0)-\omega\left(t_{0}\right)=|\beta-\alpha|$.

Case $1.0<\alpha-\beta<1 / 2$. Since $\omega(x)$ is decreasing on $(0, \infty)$, we obtain

$$
v^{\prime}(0)<0, \quad \text { for } 0<t<t_{0}
$$

and

$$
v^{\prime}(0)>0, \quad \text { for } t>t_{0}
$$

Hence (2.4) and (2.6) imply that $v^{\prime}(x)<0$ for $0<t<t_{0}$ on $(0,1)$, namely, $\phi(x)$ is decreasing from $(0,1)$ onto $\left(e^{-\alpha t}, e^{-\beta t}\right)$.

Case 2. $-1 / 2<\alpha-\beta<0$. By the same argument, assertion (4) can be proved.

Simultaneously, we observe that $v^{\prime}(1)<0$ for $t>t_{0}$. This in combination with (2.7) and $v^{\prime \prime}(x)<0$ suggests that $v^{\prime}(x)$ is strictly decreasing and therefore has a unique zero point $x_{0}(t)$, that is, $v(x)$ is increasing on $\left(0, x_{0}(t)\right)$ and decreasing on $\left(x_{0}(t), 1\right)$. Moreover, for $\alpha=$ $\beta$, it follows from (2.2) that $v^{\prime}(x)$ has a unique zero point at $x=\frac{1}{2}$. This completes the proof. 


\section{Main results}

For $x, y \in \mathbb{R}$, let

$$
\begin{aligned}
& D_{1}=\{(x, y) \mid x \leq 0, y \leq 0\} \quad \text { and } \quad D_{2}=\{(x, y) \mid x>0, y>0\}, \\
& D_{3}=\{(x, y) \mid x \geq M, y \geq M\} \cup\left\{(x, y)|| x-y \mid \geq \frac{1}{2}, x \geq 0, y \geq 0\right\},
\end{aligned}
$$

where

$$
M=\max _{x>0}\left\{G(x)=\frac{\ln x\left(1-e^{-x}\right)-2 \ln \left(1-e^{-x / 2}\right)-\ln 4}{x}\right\}<\frac{1}{2} .
$$

We point here that $M$ in (3.1) is well defined since $\lim _{x \rightarrow 0} G(x)=0$ and $\lim _{x \rightarrow \infty} G(x)=$ 0 . In fact, $G(x)$ reaches the maximum at $x_{0}=10.042944 \ldots$, that is, $M=\max _{x>0} G(x)=$ $0.09297 \ldots$

Theorem 3.1 For $\lambda, \alpha, \beta \in \mathbb{R}, \eta=\min (\alpha, \beta, 0)$ and $m, n \in \mathbb{N}$, let the function $L(x ; \lambda, \alpha, \beta)$ be defined by (1.15). Then we have

(1) For $(\alpha, \beta) \in D_{1},-L(x ; \lambda, \alpha, \beta)$ is completely monotonic on $(-\eta, \infty)$ if and only if $\lambda \geq$ $\frac{(m+n-1) !}{(m-1) !(n-1) !}$, and so is the function $L(x ; \lambda, \alpha, \beta)$ if and only if $\lambda \leq 0$;

(2) For $(\alpha, \beta) \in D_{2}, L(x ; \lambda, \alpha, \beta)$ is completely monotonic on $(0, \infty)$ if and only if $\lambda \leq$ $\inf _{t>0} 1 / W(t)$. In particular, if $(\alpha, \beta) \in D_{3}$, then $\inf _{t>0} 1 / W(t)=\frac{(m+n-1) !}{(m-1) !(n-1) !}$, where

$$
W(t)=\int_{0}^{1} \frac{t\left(1-e^{-t}\right)}{1-e^{-t x}} \frac{x^{m}(1-x)^{n}}{1-e^{-t(1-x)}} e^{-\alpha t x} e^{-\beta t(1-x)} d x, \quad t>0 .
$$

Proof Using the well-known formula (1.2) and applying the convolution theorem for the Laplace transform, we have

$$
L(x ; \lambda, \alpha, \beta)=\int_{0}^{\infty} e^{-x t} P(t) d t,
$$

where

$$
P(t)=\int_{0}^{t}\left(\frac{t^{m+n-1}}{1-e^{-t}}-\lambda \frac{s^{m}}{1-e^{-s}} \frac{(t-s)^{n}}{1-e^{-(t-s)}} e^{-\alpha s} e^{-\beta(t-s)}\right) d s .
$$

Changing of variable $s=t x$ yields

$$
P(t)=\frac{t^{m+n} \lambda}{1-e^{-t}}\left(\frac{1}{\lambda}-W(t)\right)
$$

Using the integral representation

$$
\int_{0}^{1} x^{m}(1-x)^{n} d x=\frac{n ! m !}{(n+m+1) !}
$$

for $\lambda=\frac{(m+n-1) !}{(m-1) !(n-1) !}$, the expression (3.3) can be written as

$$
P(t)=\frac{\lambda t^{m+n}}{1-e^{-t}} \int_{0}^{1} x^{m-1}(1-x)^{n-1} U(x) d x,
$$


where

$$
U(x)=1-\frac{t\left(1-e^{-t}\right)}{1-e^{-t x}} \frac{x(1-x)}{1-e^{-t(1-x)}} e^{-\alpha t x} e^{-\beta t(1-x)} .
$$

Case 1. $(\alpha, \beta) \in D_{1}$. First of all, we shall show that

$$
1<\frac{t\left(1-e^{-t}\right)}{1-e^{-t x}} \frac{x(1-x)}{1-e^{-t(1-x)}}, \quad \text { for } t>0 \text { and } 0<x<1,
$$

which is equivalent to

$$
V(t)=\left(1-e^{-t x}\right)\left(1-e^{-t(1-x)}\right)-x(1-x) t\left(1-e^{-t}\right)<0 .
$$

A simple computation gives $e^{t} V^{\prime}(t)=V_{1}(t)$ and

$$
V_{1}^{\prime}(t)=x(1-x) \sum_{k=1}^{\infty} \frac{t^{k}}{k !}\left(x^{k}+(1-x)^{k}-1\right)<0,
$$

which together with $V_{1}(0)=0$ yields that $V_{1}(t)<0$ for $t>0$ and $0<x<1$. Furthermore, combining this with $V(0)=0$ and $e^{t} V^{\prime}(t)=V_{1}(t)$, we have $V(t)<0$. Hence for $(\alpha, \beta) \in D_{1}$, we see that

$$
1<\frac{t\left(1-e^{-t}\right)}{1-e^{-t x}} \frac{x(1-x)}{1-e^{-t(1-x)}} e^{-\alpha t x} e^{-\beta t(1-x)}, \quad \text { for } t>0 \text { and } 0<x<1,
$$

that is $U(x)>0$ for $t>0$ and $0<x<1$.

From (3.2), (3.4) and (3.6), we conclude that $W(t)>\frac{(m-1) !(n-1) !}{(m+n-1) !}$ for $t>0$. For $(\alpha, \beta) \in D_{1}$, we also observe that $\lim _{t \rightarrow 0} W(t)=\frac{(m-1) !(n-1) !}{(m+n-1) !}$, and $\lim _{t \rightarrow \infty} W(t)=+\infty$. Hence we have the sharp inequality

$$
0<\frac{1}{W(t)}<\frac{(m+n-1) !}{(m-1) !(n-1) !}, \quad \text { for all } t>0 .
$$

Finally, according to (3.3), (3.7) and the Bernstein-Widder Theorem, we complete the proof of assertion (1).

Case 2. $(\alpha, \beta) \in D_{2}$. Since $\lim _{t \rightarrow 0} W(t)=\frac{(m-1) !(n-1) !}{(m+n-1) !}$ and $\lim _{t \rightarrow \infty} W(t)=0$, then $\lambda \leq$ $\inf _{t>0} 1 / W(t)$ is well defined. Once more using the Bernstein-Widder Theorem and (3.3), we know that $L(x ; \lambda, \alpha, \beta)$ is completely monotonic on $(0, \infty)$ if and only if $\lambda \leq$ $\inf _{t>0} 1 / W(t)$.

In particular, we consider the case $(\alpha, \beta) \in D_{3}$. If we prove

$$
1 \geq \frac{t\left(1-e^{-t}\right)}{1-e^{-t x}} \frac{x(1-x)}{1-e^{-t(1-x)}} e^{-\alpha t x} e^{-\beta t(1-x)}, \quad t>0,0<x<1,
$$

we get $\inf _{t>0} 1 / W(t)=\frac{(m+n-1) !}{(m-1) !(n-1) !}$ according to (3.2), (3.4) and $\lim _{t \rightarrow 0} W(t)=\frac{(m-1) !(n-1) !}{(m+n-1) !}$.

For $\alpha=\beta \geq M, U(x)$ is reduced to

$$
U_{1}(x)=1-\frac{t\left(1-e^{-t}\right)}{1-e^{-t x}} \frac{x(1-x)}{1-e^{-t(1-x)}} e^{-\alpha t} .
$$


In virtue of Lemma 1 , we know that $U_{1}(x)$ is decreasing on $(0,1 / 2)$ and increasing on $(1 / 2,1)$, that is, $U_{1}(x) \geq U_{1}\left(\frac{1}{2}\right)$. Since $\alpha \geq M$ is equivalent to $U_{1}\left(\frac{1}{2}\right) \geq 0$, we have $U_{1}(x) \geq 0$ for $t>0$ and $0<x<1$.

If $\beta \geq M$ and $\alpha \geq \beta$, then we write

$$
U(x)=1-\frac{t\left(1-e^{-t}\right)}{1-e^{-t x}} \frac{x(1-x)}{1-e^{-t(1-x)}} e^{-\beta t} e^{(\beta-\alpha) t x} .
$$

Together with $U_{1}(x) \geq 0$, it leads to (3.8). Similarly, we can prove that (3.8) is still valid for the case $\alpha \geq M$ and $\beta \geq \alpha$.

If $\beta-\alpha \geq \frac{1}{2}$ and $\alpha \geq 0$ or $\beta-\alpha \leq-\frac{1}{2}$ and $\beta \geq 0$, in view of Lemma 1 , we can prove (3.8). The proof is completed.

Remark 1 Obviously, Theorem 3.1 is a generalization of [28, Theorem 3] for higher derivatives of $\psi(x)$.

Corollary 1 For $\alpha \in \mathbb{R}, m, n \in \mathbb{N}$ and $\lambda=\frac{(m+n-1) !}{(m-1) !(n-1) !}$, the functions

$$
\begin{aligned}
& f_{1}(x)=\lambda \psi_{n}(x+\alpha) \psi_{m}(x+\alpha)-\psi_{m+n}(x), \\
& f_{2}(x)=\lambda \psi_{n}(x) \psi_{m}(x+\alpha)-\psi_{m+n}(x)
\end{aligned}
$$

are completely monotonic on $(-\alpha, \infty)$ if and only if $\alpha \leq 0$.

Proof The sufficient conditions of the assertion is proved in the proof of Theorem 3.1.

Next we shall prove the necessary conditions.

Suppose that $\alpha>0$. Since $f_{1}(x)$ and $f_{2}(x)$ are completely monotonic on $(0, \infty)$, we have $f_{1}(x), f_{2}(x) \geq 0$. On the other hand, it is easy to check that

$$
\begin{aligned}
& \lim _{x \rightarrow 0+} f_{1}(x)=\lambda \psi_{m}(\alpha) \psi_{n}(\alpha)-\lim _{x \rightarrow 0+} \psi_{m+n}(x)=-\infty, \\
& \lim _{x \rightarrow 0+} \frac{f_{2}(x)}{\psi_{n}(x)}=\lambda \psi_{n}(\alpha)-\lim _{x \rightarrow 0+} \frac{\psi_{m+n}(x)}{\psi_{m}(x)}=-\infty
\end{aligned}
$$

which yields contradictions.

The proof is completed.

Remark 2 The function $f_{1}(x)$ can be written equivalently as $h_{1}(t)=f_{1}(t-\alpha)(t>0)$. By hypothesis, we get

$$
\alpha \leq t-\psi_{m+n}^{-1}\left[\lambda \psi_{n}(t) \psi_{m}(t)\right]
$$

which yields $\alpha \leq 0$ as $t \rightarrow 0$. Furthermore, Corollary 1 clearly strengthens [28, Theorem 1 ].

Remark 3 In [27, Theorem 2.2], Batir proved the inequality

$$
\left(\frac{\psi_{n}(x+1 / 2)}{(n-1) !}\right)^{\frac{m}{n}}<\frac{\psi_{m}(x)}{(m-1) !}<\left(\frac{\psi_{n}(x)}{(n-1) !}\right)^{\frac{m}{n}}
$$


for $m \in \mathbb{N}, n=1,2, \ldots, m-1$ and $x>0$. By Theorem 3.1, inequality (3.11) can be refined partially. Taking the logarithm in (3.11) yields

$$
\frac{1}{n} \ln \left(\frac{\psi_{n}(x+1 / 2)}{(n-1) !}\right)<\frac{1}{m} \ln \left(\frac{\psi_{m}(x)}{(m-1) !}\right)<\frac{1}{n} \ln \left(\frac{\psi_{n}(x)}{(n-1) !}\right) .
$$

Corollary 2 For $m$ and $n \in \mathbb{N}$, we have the following inequalities

$$
\begin{aligned}
\left(\frac{\psi_{n}(x+1 / 2)}{(n-1) !}\right)^{\frac{m+n}{n}} & <\frac{\psi_{m}(x)}{(m-1) !} \frac{\psi_{n}(x+1 / 2)}{(n-1) !}<\frac{\psi_{m+n}(x)}{(m+n-1) !} \\
& <\frac{\psi_{m}(x)}{(m-1) !} \frac{\psi_{n}(x)}{(n-1) !}<\left(\frac{\psi_{n}(x)}{(n-1) !}\right)^{\frac{m+n}{n}}, \quad(m>n), \\
\left(\frac{\psi_{n}(x+M)}{(n-1) !}\right)^{\frac{m+n}{n}} & <\frac{\psi_{m}(x+M)}{(m-1) !} \frac{\psi_{n}(x+M)}{(n-1) !}<\frac{\psi_{m+n}(x)}{(m+n-1) !} \\
& <\left(\frac{\psi_{n}(x)}{(n-1) !}\right)^{\frac{m+n}{n}}<\frac{\psi_{m}(x)}{(m-1) !} \frac{\psi_{n}(x)}{(n-1) !}, \quad(m<n)
\end{aligned}
$$

for $x>0$, where $M$ is defined by (3.1).

Proof On the one hand, if $m>n$, a simple calculation shows that the right-hand side of (3.12) is equivalent to

$$
\frac{\psi_{m}(x)}{(m-1) !} \frac{\psi_{n}(x)}{(n-1) !}<\left(\frac{\psi_{n}(x)}{(n-1) !}\right)^{\frac{m+n}{n}} .
$$

Similarly, the left-hand side of (3.12) is equivalent to

$$
\left(\frac{\psi_{n}(x+1 / 2)}{(n-1) !}\right)^{\frac{m+n}{n}}<\frac{\psi_{m}(x)}{(m-1) !} \frac{\psi_{n}(x+1 / 2)}{(n-1) !} .
$$

By (3.15), (3.16) and Theorem 3.1, we see that (3.13) is proved.

On the other hand, if $m<n$, inequality (3.15) is reversed by a similar calculation. From the reversed inequality of (3.15), it follows that

$$
\left(\frac{\psi_{n}(x+M)}{(n-1) !}\right)^{\frac{m+n}{n}}<\frac{\psi_{m}(x+M)}{(m-1) !} \frac{\psi_{n}(x+M)}{(n-1) !} .
$$

Taking into account the right-hand side of (3.12), the reversed inequality of (3.15), (3.17), and Theorem 3.1, we prove (3.14).

Consequently, the proof of the two inequalities is complete.

\section{Application}

In [36], Elezović et al. derived that

$$
\psi_{1}(x)<e^{-\psi(x)}
$$

by the fact that the function $e^{\psi(x+t)}-x$ is decreasing on $(0, \infty)$ for all $t>0$. In addition, [26, Lemma 1.2] provides a different proof of (4.1). Some extensions of (4.1) for higher-order 
derivatives of $\psi(x)$ can be found in [25, 27]. For example, it is given in [27, Theorem 2.1] that the inequality (4.1) was generalized to

$$
e^{-n \psi(x+\beta)}<\frac{\psi_{n}(x)}{(n-1) !}<e^{-n \psi(x+\alpha)}
$$

for $x>0$ and $n \in \mathbb{N}$, where $\beta=1 / 2$ and $\alpha=0$. In particular, inequality (4.2) was proved again by using monotonicity of functions involving the polygamma functions (see [37, Corollary 1]).

We introduce the divided differences of psi and polygamma functions (see [38]). For $n \in \mathbb{N}, s, t \in \mathbb{R}, r=\min \{s, t\}$ and $x \in(-r, \infty)$, we define

$$
\phi_{n}(x)= \begin{cases}\frac{\psi_{n-1}(x+s)-\psi_{n-1}(x+t)}{t-s}, & t \neq s ; \\ \psi_{n}(x+t), & t=s .\end{cases}
$$

For the sake of consistency, we set $\psi_{0}(x)=-\psi(x)$.

Using Theorem 3.1 and inequality (4.2), we establish the following result.

Corollary 3 For $\beta \in \mathbb{R}$ and $n \in \mathbb{N}$, let the function $f_{3}(x)=(n-1) ! e^{-n \psi(x+\beta)}-\psi_{n}(x)$ be defined on $(\max (-\beta, 0), \infty)$. Then the function $f_{3}(x)$ is decreasing on $(-\beta, \infty)$ if $\beta \leq 0$; and is increasing on $(0, \infty)$ if $\beta \geq \frac{1}{2}$.

Proof A simple computation gives

$$
f_{3}^{\prime}(x)=\psi_{n+1}(x)-n(n-1) ! \psi_{1}(x+\beta) e^{-n \psi(x+\beta)} .
$$

For $\beta \leq 0$, from the right-hand side of (4.2), we get

$$
f_{3}^{\prime}(x)<\psi_{n+1}(x)-n \psi_{1}(x+\beta) \psi_{n}(x+\beta),
$$

and therefore, in the view of Theorem 3.1, we have $f_{3}^{\prime}(x)<0$. By the same spirit, the lefthand side of (4.2) and Theorem 3.1 imply the case $\beta \geq \frac{1}{2}$.

This completes the proof.

Remark 4 For $\lambda \neq 0, s, t \in \mathbb{R}$ and $r=\min \{s, t\}$, define the function $\Psi$ for $x \in(-r, \infty)$

$$
\Psi(x ; \lambda, s, t)= \begin{cases}{\left[\frac{\Gamma(x+t)}{\Gamma(x+s)}\right]^{\frac{1}{\lambda(t-s)}},} & t \neq s ; \\ e^{\frac{1}{\lambda} \psi(x+s)}, & t=s .\end{cases}
$$

It was shown in [36] that the function $\Psi(x ; 1, s, t)$ is convex on $(-r, \infty)$ for $|t-s|<1$ and concave on the same interval for $|t-s|>1$. Since

$$
\Psi^{\prime \prime}(x ; \lambda, s, t)=\frac{1}{\lambda^{2}} \Psi(x ; \lambda, s, t)\left(\phi_{1}^{2}(x)-\lambda \phi_{2}(x)\right),
$$

we deduce from [39, Theorem 3.1]:

(1) For $0<|t-s|<1$, the function $\Psi(x ; \lambda, s, t)$ is convex on $(-r, \infty)$ if and only if $\lambda \neq 0 \leq 1$ and concave on the same interval if and only if $\lambda \geq \frac{1}{|t-s|}$; 
(2) For $|t-s|>1$, the function $\Psi(x ; \lambda, s, t)$ is convex on $(-r, \infty)$ if and only if $\lambda \neq 0 \leq \frac{1}{|t-s|}$ and concave on the same interval if and only if $\lambda \geq 1$;

(3) For $|t-s|=1$, the function $\Psi(x ; \lambda, s, t)$ is convex on $(-r, \infty)$ if and only if $\lambda \neq 0 \leq 1$ and concave on the same interval if and only if $\lambda \geq 1$;

(4) For $s=t$, the function $\Psi(x ; \lambda, s, t)$ is convex on $(-r, \infty)$ if and only if $\lambda \neq 0 \leq 1$.

In addition, it was proved in [36] that

$$
\Psi(x ; 1, s, t) \phi_{1}(x)<1
$$

holds for $x>-r$ if $|t-s|<1$ and its reversed inequality is valid on $(-r, \infty)$ if $|t-s|>1$. Obviously, (4.5) is a generalization of (4.1).

In the following, we will prove the monotonicity of the function $z(x ; \lambda, s, t)=\Psi(x ; \lambda, s, t) \times$ $\phi_{n}(x)$ and therefore extend (4.5) or the right-hand side of (4.2).

Theorem 4.1 For $\lambda \neq 0, s, t \in \mathbb{R}, r=\min (s, t)$ and $n \in \mathbb{N}$, the function $z(x ; \lambda, s, t)$ has the following monotonic properties:

(1) For $0<|t-s|<1$, the function $z(x ; \lambda, s, t)$ is increasing on $(-r, \infty)$ if and only if $1 / \lambda \geq n$ and decreasing on the same interval if and only if $1 / \lambda \leq n|t-s|$;

(2) For $|t-s|>1$, the function $z(x ; \lambda, s, t)$ is increasing on $(-r, \infty)$ if and only if $1 / \lambda \geq n|t-s|$ and decreasing on the same interval if and only if $1 / \lambda \leq n$;

(3) For $|t-s|=1$, the function $z(x ; \lambda, s, t)$ is increasing on $(-r, \infty)$ if and only if $1 / \lambda \geq n$ and decreasing on the same interval if and only if $1 / \lambda \leq n$;

(4) For $s=t$, the function $z(x ; \lambda, s, t)$ is increasing on $(-r, \infty)$ if and only if $1 / \lambda \geq n$ and decreasing on the same interval if and only if $1 / \lambda \leq 0$.

Proof Differentiating $z(x ; \lambda, s, t)$ yields

$$
z^{\prime}(x ; \lambda, s, t)=\Psi(x ; \lambda, s, t)\left(\frac{1}{\lambda} \phi_{1}(x) \phi_{n}(x)-\phi_{n+1}(x)\right) .
$$

This in combination with Theorem [39, Theorem 3.1] easily establishes the Theorem.

Using Theorem 4.1, we have the following:

Corollary 4 For $s, t \in \mathbb{R}, r=\min \{s, t\}$ and $n \in \mathbb{N}$, we have the inequality

$$
\Psi\left(x ; \frac{1}{n}, s, t\right) \phi_{n}(x)<(n-1) !
$$

for $x>-r$ if $|t-s|<1$ and its reversed inequality is valid on $(-r, \infty)$ if $|t-s|>1$.

Proof Obviously, we only assume $s \neq t$. In view of Theorem 4.1, we only need to check

$$
\lim _{x \rightarrow \infty} \Psi\left(x ; \frac{1}{n}, s, t\right) \phi_{n}(x)=(n-1) !
$$

Applying the asymptotic formula (1.5), we obtain

$$
\lim _{x \rightarrow \infty} \frac{e^{n \psi(x)}}{x^{n}}=1
$$


Therefore, this together with [24, Lemma 4] establishes

$$
\lim _{x \rightarrow \infty} e^{n \psi(x+c)} \phi_{n}(x)=(n-1) !, \quad \text { for all } c \in \mathbb{R}
$$

According to [13, Corollary 1.4], the inequality

$$
e^{\psi(x+r)}<\left[\frac{\Gamma(x+t)}{\Gamma(x+s)}\right]^{1 /(t-s)}<e^{\psi\left(x+\frac{s+t}{2}\right)}
$$

holds for $x>-r$, so that this combined with (4.8) yields (4.7).

Hence we complete the proof of this Theorem.

Theorem 4.2 For $s, t \in \mathbb{R}, r=\min \{s, t\}$ and $c \in(-r, \infty)$, we have the double inequality

$$
\frac{e^{G_{s, t}\left(X_{S, t}\right)}}{e^{H_{s, t}\left(X_{S, t}\right)}}<\frac{e^{G_{s, t}(x)}}{e^{H_{s, t}(x)}}<\frac{\sqrt{2 \pi e} e^{A_{c, s, t}-(s+t) / 2}}{\Gamma\left(c+\frac{s+t}{2}\right)}
$$

for $x>X_{s, t}$ if $|t-s|<1$ and its reversed inequality is valid on $\left(X_{s, t}, \infty\right)$ if $|t-s|>1$, where $X_{s, t}$ is the only zero of $1+\ln \Psi(x ; 1, s, t)$ on $(-r, \infty)$,

$$
\begin{aligned}
& G_{s, t}(x)= \begin{cases}\frac{1}{t-s} \int_{c}^{x} \ln \left[\frac{\Gamma(u+t)}{\Gamma(u+s)}\right] d u, & t \neq s, \\
\int_{c}^{x} \psi(u+s) d u, & t=s ;\end{cases} \\
& A_{c, s, t}= \begin{cases}\int_{c}^{\infty} \frac{1}{t-s} \ln \left[\frac{\Gamma(u+t)}{\Gamma(u+s)}\right]-\psi\left(u+\frac{s+t}{2}\right) d u, & t \neq s, \\
0, & t=s ;\end{cases}
\end{aligned}
$$

and

$$
H_{s, t}(x)=\Psi(x ; 1, s, t) \ln \Psi(x ; 1, s, t)-x .
$$

Proof Let $g_{s, t}(x)=e^{G_{s, t}(x)}, h_{s, t}(x)=\ln \Psi(x ; 1, s, t)$ and $f_{s, t}(x)=g_{s, t}(x) e^{x-h_{s, t}(x) e^{h_{s, t}(x)}}$. Since $g_{s, t}^{\prime}(x)=g_{s, t}(x) h_{s, t}(x)$ and $h_{s, t}^{\prime}(x)=\phi_{1}(x)$, we obtain

$$
f_{s, t}^{\prime}(x)=g_{s, t}(x) e^{x-h_{s, t}(x) e^{h_{s, t}(x)}}\left(1-\phi_{1}(x) e^{h_{s, t}(x)}\right)\left(1+h_{s, t}(x)\right) .
$$

Using the asymptotic formula (see [4])

$$
\frac{\Gamma(x+t)}{\Gamma(x+s)}=x^{t-s}\left(1-\frac{(s-t)(s+t-1)}{2 x}+O\left(\frac{1}{x^{2}}\right)\right), \quad x \rightarrow \infty,
$$

we get $\lim _{x \rightarrow \infty} h_{s, t}(x)=\infty$, and therefore by $h_{s, t}^{\prime}(x)>0$ and $\lim _{x \rightarrow-r} h_{s, t}(x)=-\infty$, we conclude that $1+h_{s, t}(x)$ has a unique zero on $(-r, \infty)$.

Hence thanks to $h_{s, t}^{\prime}(x)>0$, Corollary 4 and (4.11), we have the following statements:

(i) For $|t-s|<1, f_{s, t}(x)$ is increasing on $\left(X_{s, t}, \infty\right)$ and decreasing on $\left(-r, X_{s, t}\right)$,

(ii) For $|t-s|>1, f_{s, t}(x)$ is decreasing on $\left(X_{s, t}, \infty\right)$ and increasing on $\left(-r, X_{s, t}\right)$. 
On the one hand, we check that

$$
\begin{gathered}
\lim _{x \rightarrow \infty} \int_{c}^{x} \psi\left(u+\frac{s+t}{2}\right) d u+x-h_{s, t}(x) e^{h_{s, t}(x)} \\
\quad=\frac{1}{2}+\frac{1}{2} \ln (2 \pi)-\frac{s+t}{2}-\ln \Gamma\left(c+\frac{s+t}{2}\right) .
\end{gathered}
$$

Case 1. $s \neq t$. Now we derive the asymptotic formula of $h_{s, t}(x) e^{h_{s, t}(x)}$. Taking the logarithm in (4.12), we get

$$
\begin{aligned}
h_{s, t}(x) & =\frac{[\ln \Gamma(x+t)-\ln \Gamma(x+s)]}{t-s} \\
& =\ln x+\frac{1}{t-s} \ln \left(1-\frac{(s-t)(s+t-1)}{2 x}+O\left(\frac{1}{x^{2}}\right)\right), \quad x \rightarrow \infty .
\end{aligned}
$$

Together with

$$
\ln (1+x)=x-\frac{x^{2}}{2}+O\left(x^{3}\right), \quad x \rightarrow 0,
$$

we can rewrite (4.14) as

$$
\begin{aligned}
h_{s, t}(x) & =\frac{[\ln \Gamma(x+t)-\ln \Gamma(x+s)]}{t-s} \\
& =\ln x+\frac{t+s-1}{2 x}+O\left(\frac{1}{x^{2}}\right), \quad x \rightarrow \infty,
\end{aligned}
$$

which implies that

$$
h_{s, t}(x) e^{h_{s, t}(x)}=x\left(\ln x+\frac{t+s-1}{2 x}+O\left(\frac{1}{x^{2}}\right)\right) e^{\frac{t+s-1}{2 x}+O\left(\frac{1}{x^{2}}\right)}, \quad x \rightarrow \infty .
$$

Therefore, by the aid of

$$
e^{x}=1+x+O\left(x^{2}\right), \quad x \rightarrow 0,
$$

we obtain

$$
\begin{aligned}
h_{s, t}(x) e^{h_{s, t}(x)}= & x \ln x+\frac{t+s-1}{2} \ln x+\frac{t+s-1}{2}+\frac{(t+s-1)^{2}}{4 x} \\
& +O\left(\frac{\ln x}{x}\right)+O\left(\frac{1}{x^{2}}\right), \quad x \rightarrow \infty
\end{aligned}
$$

Combining (1.4) with (4.17), we deduce that

$$
\begin{aligned}
\ln \Gamma\left(x+\frac{s+t}{2}\right)+x-h_{s, t}(x) e^{h_{s, t}(x)} \\
=x \ln \left(1+\frac{s+t}{2 x}\right)+\frac{t+s-1}{2} \ln \left(1+\frac{s+t}{2 x}\right) \\
\quad+\frac{1}{2}-(t+s)+\frac{1}{2} \ln (2 \pi)+\frac{1}{12} \frac{1}{x+\frac{s+t}{2}}
\end{aligned}
$$




$$
-\frac{(t+s-1)^{2}}{4 x}+O\left(\frac{\ln x}{x}\right)+O\left(\frac{1}{x^{2}}\right), \quad x \rightarrow \infty
$$

which implies (4.13).

Case 2. $s=t$. Using (1.4) and the asymptotic formula (see [4])

$$
\psi\left(x+\frac{s+t}{2}\right)=\ln x+\frac{s+t-1}{2 x}+O\left(\frac{1}{x^{2}}\right), \quad x \rightarrow \infty
$$

we can easily prove (4.13).

On the other hand, we show that

$$
\lim _{x \rightarrow \infty}\left(G_{s, t}(x)-\int_{c}^{x} \psi\left(u+\frac{s+t}{2}\right) d u\right)= \begin{cases}A_{c, s, t}, & t \neq s \\ 0, & t=s\end{cases}
$$

where

$$
\int_{c}^{\infty} \frac{1}{t-s} \ln \left[\frac{\Gamma(u+t)}{\Gamma(u+s)}\right]-\psi\left(u+\frac{s+t}{2}\right) d u=A_{c, s, t} .
$$

Note that the case $t=s$ is obvious. Then using (4.15) and (4.19), we get

$$
\frac{1}{t-s} \ln \left[\frac{\Gamma(x+t)}{\Gamma(x+s)}\right]-\psi\left(x+\frac{s+t}{2}\right)=O\left(\frac{1}{x^{2}}\right), \quad x \rightarrow \infty
$$

which implies the exitance of constants $C$ and $X>0$ such that

$$
\left|\frac{1}{t-s} \ln \left[\frac{\Gamma(x+t)}{\Gamma(x+s)}\right]-\psi\left(x+\frac{s+t}{2}\right)\right| \leq C\left|\frac{1}{x^{2}}\right|
$$

for all $x>X$. It follows that

$$
\lim _{x \rightarrow \infty} \int_{x}^{\infty} \frac{1}{t-s} \ln \left[\frac{\Gamma(u+t)}{\Gamma(u+s)}\right]-\psi\left(u+\frac{s+t}{2}\right) d u=0
$$

so that $A_{c, s, t}$ is well defined. Hence, (4.20) is proved.

Finally, taking into consideration (4.13) and (4.20), we have

$$
\begin{aligned}
\lim _{x \rightarrow \infty}\left(G_{s, t}(x)+x-h_{s, t}(x) e^{h_{s, t}(x)}\right) \\
\quad=\left\{\begin{array}{l}
\frac{1}{2}+\frac{1}{2} \ln (2 \pi)-\frac{s+t}{2}-\ln \Gamma\left(c+\frac{s+t}{2}\right)+A_{c, s, t}, \quad t \neq s ; \\
\frac{1}{2}+\frac{1}{2} \ln (2 \pi)-s-\ln \Gamma(c+s), \quad t=s .
\end{array}\right.
\end{aligned}
$$

Applying the monotonicity of $f_{s, t}(x)$ and (4.21), we complete the proof of this Theorem.

Remark 5 Let $0.785003 \leq s<t$. Using the inequality (see [13, Corollary 1.4])

$$
\psi(x+s)<\frac{1}{t-s}[\ln \Gamma(x+t)-\ln \Gamma(x+s)]<\psi\left(x+\frac{s+t}{2}\right), \quad x>-s,
$$


we have $1+h_{s, t}(0)>1+\psi(s) \geq 0$, so that by $h_{s, t}^{\prime}(x)>0$, Corollary 4 and (4.11) again, we conclude that $f_{s, t}(x)$ is increasing on $(0, \infty)$ if $|t-s|<1$ and decreasing on the same interval if $|t-s|>1$. Similarly, we have the inequality

$$
\begin{aligned}
{\left[\frac{\Gamma(x+t)}{\Gamma(x+s)}\right]^{\frac{1}{t-s}} \ln \left[\frac{\Gamma(x+t)}{\Gamma(x+s)}\right]^{\frac{1}{t-s}}<} & {\left[\frac{\Gamma(t)}{\Gamma(s)}\right]^{\frac{1}{t-s}} \ln \left[\frac{\Gamma(t)}{\Gamma(s)}\right]^{\frac{1}{t-s}}+x } \\
& +\frac{1}{t-s} \int_{0}^{x} \ln \left[\frac{\Gamma(u+t)}{\Gamma(u+s)}\right] d u
\end{aligned}
$$

for $x>0$ if $|t-s|<1$ and its reversed inequality is valid on $(0, \infty)$ if $|t-s|>1$.

\section{Discussion}

Observing that Corollary 4 generalizes the right-hand side of (4.2), we conjecture that the left-hand side of (4.2) might be generalized to

$$
(n-1) !<\Psi\left(x+\frac{1}{2} ; \frac{1}{n}, s, t\right) \phi_{n}(x)
$$

for $x>-r$ if $|t-s|<1$ and that its reversed inequality might be valid on $(-r, \infty)$ if $|t-s|>1$, where $s, t \in \mathbb{R}$ and $r=\min \{s, t\}$.

We turn to pay attention to the class of strongly completely monotonic functions, which are introduced in [40]. A function $f:(0, \infty) \rightarrow \mathbb{R}$ is called strongly completely monotonic if it satisfies the more restrictive condition that $(-1)^{n} x^{n+1} f^{(n)}(x)$ is nonnegative and decreasing on $(0, \infty)$ for all $n \in \mathbb{N}$. Note that $[40$, Theorem 1] gives a characterization of strongly completely monotonic functions.

It was shown in [20] that the function $\psi_{1}^{2}(x)-\psi_{2}(x)$ is strongly completely monotonic on $(0, \infty)$. Inspired by this, we will determine necessary and sufficient conditions for $\lambda$ such that the function $\Phi(x ; \lambda, s, t)$ is strongly completely monotonic on $(-r, \infty)$ for all fixed $s, t \in \mathbb{R}$ and $r=\min \{s, t\}$ in the future work.

\section{Acknowledgements}

The authors would like to express their sincere thanks to the editors and reviewers for their great efforts to improve this paper.

\section{Funding}

Research was supported by the National Natural Science Foundation of China (No. 12061033), Inner Mongolia Natural Science Foundation (No. 2018MS01023), Natural Science Basic Research Plan of Shaanxi Province (2020JM-175) and Teaching Reform Project of Northwest A\&F University (JY1902013, JXGG2130).

Availability of data and materials

Not applicable.

\section{Declarations}

Competing interests

The authors declare that they have no competing interests.

\section{Authors' contributions}

All authors contributed to each part of this work equally, and they read and approved the final manuscript.

\section{Author details}

${ }^{1}$ College of Science, Northwest A\&F University, Yangling, Shaanxi, 712100, P.R. China. ${ }^{2}$ Department of Mathematics, Hulunbeir College, Hailar, Inner Mongolia, 021008, P.R. China. 


\section{Publisher's Note}

Springer Nature remains neutral with regard to jurisdictional claims in published maps and institutional affiliations.

\section{Received: 9 April 2021 Accepted: 22 December 2021 Published online: 07 January 2022}

\section{References}

1. Davis, P.J.: Leonhard Euler's integral: a historical profile of the gamma function. Am. Math. Mon. 66, 849-869 (1959)

2. Abramowitz, M., Stegun, I.A.: Handbook of Mathematical Functions with Formulas, Graphs, and Mathematical Tables, 10th printing edn. National Bureau of Standards Applied Mathematics Series, vol. 55. Dover, New York (1972)

3. Magnus, W., Oberhettinger, F., Soni, R.P.: Formulas and Theorems for the Special Functions of Mathematical Physics. Springer, Berlin (1966)

4. Luke, Y.L.: The Special Functions and Their Approximations. Vol. II. Mathematics in Science and Engineering, vol. 53. Academic Press, New York (1969)

5. Andrews, G.E., Askey, R., Roy, R.: Special Functions. Cambridge University Press, Cambridge (1999)

6. Alzer, H.: Mean-value inequalities for the polygamma functions. Aequ. Math. 61(1-2), 151-161 (2001). https://doi.org/10.1007/s000100050167

7. Batir, N.: An interesting double inequality for Euler's gamma function. J. Inequal. Pure Appl. Math. 5(4), 97 (2004). http://www.emis.de/journals/JPAM/article452.html

8. Alzer, H.: On some inequalities for the gamma and psi functions. Math. Compet. 66(217), 373-389 (1997). https://doi.org/10.1090/S0025-5718-97-00807-7

9. Alzer, H., Wells, J.: Inequalities for the polygamma functions. SIAM J. Math. Anal. 29, 1459-1466 (1998). https://doi.org/10.1137/S0036141097325071

10. Alzer, H.: A power mean inequality for the gamma function. Monatshefte Math. 131(3), 179-188 (2000). https://doi.org/10.1007/s006050070007

11. Yang, Z.H., Zheng, S.Z.: Monotonicity of a mean related to polygamma functions with an application. J. Inequal. Appl. 2016(1), 216 (2016). https://doi.org/10.1186/s13660-016-1155-4

12. Guo, B.-N., Qi, F.: Refinements of lower bounds for polygamma functions. Proc. Am. Math. Soc. 141(3), 1007-1015 (2013). https://doi.org/10.1090/S0002-9939-2012-11387-5

13. Elezović, N., Pečarić, J.: Differential and integral $f$-means and applications to digamma function. Math. Inequal. Appl. 3(2), 189-196 (2000). https://doi.org/10.7153/mia-03-22

14. Bustoz, J., Ismail, M.E.H.: On gamma function inequalities. Math. Compet. 47(176), 659-667 (1986). https://doi.org/10.2307/2008180

15. Gautschi, W.: Some elementary inequalities relating to the gamma and incomplete gamma function. J. Math. Phys. 38, 77-81 (1959). https://doi.org/10.1002/sapm195938177

16. Anderson, G.D., Qiu, S.L.: A monotonicity property of the gamma function. Proc. Am. Math. Soc. 125(11), 3355-3362 (1997). https://doi.org/10.1090/S0002-9939-97-04152-X

17. Widder, D.V.: The Laplace Transform. Princeton University Press, Princeton (1946)

18. Qi, F., Guo, S., Guo, B.-N.: Complete monotonicity of some functions involving polygamma functions. J. Comput. Appl. Math. 233(9), 2149-2160 (2010). https://doi.org/10.1016/j.cam.2009.09.044

19. Guo, B.-N., Qi, F., Srivastava, H.M.: Some uniqueness results for the non-trivially complete monotonicity of a class of functions involving the polygamma and related functions. Integral Transforms Spec. Funct. 21(11), 103-111 (2010). https://doi.org/10.1080/10652461003748112

20. Koumandos, S.: Monotonicity of some functions involving the gamma and psi functions. Math. Compet. 77(264), 2261-2275 (2008). https://doi.org/10.1090/S0025-5718-08-02140-6

21. Alzer, H., Berg, C.: Some classes of completely monotonic functions II. Ramanujan J. 11(2), 225-248 (2006). https://doi.org/10.1007/s11139-006-6510-5

22. Guo, B.-N., Chen, R.-J., Qi, F.: A class of completely monotonic functions involving the polygamma functions. J. Math. Anal. Approx. Theory 1(2), 124-134 (2006). https://doi.org/10.1080/23311835.2014.982896

23. Yang, Z.-H.: Approximations for certain hyperbolic functions by partial sums of their Taylor series and completely monotonic functions related to gamma function. J. Math. Anal. Appl. 441, 549-564 (2016). https://doi.org/10.1016/j.jmaa.2016.04.029

24. Yang, Z.-H.: Some properties of the divided difference of psi and polygamma functions. J. Math. Anal. Appl. 455, 761-777 (2017). https://doi.org/10.1016/j.jmaa.2017.05.081

25. Alzer, H.: Sharp inequalities for the digamma and polygamma functions. Forum Math. 16, 181-221 (2004) https://doi.org/10.1515/form.2004.009

26. Batir, N.: Some new inequalities for gamma and polygamma functions. J. Inequal. Pure Appl. Math. 6(4), 103 (2005). http://www.emis.de/journals/JPAM/article577.html

27. Batir, N.: On some properties of digamma and polygamma functions. J. Math. Anal. Appl. 328(1), 452-465 (2007). https://doi.org/10.1016/j.jmaa.2006.05.065

28. Qi, F., Guo, B.-N.: Necessary and sufficient conditions for functions involving the tri- and tetra-gamma functions to be completely monotonic. Adv. Appl. Math. 44(1), 71-83 (2010). https://doi.org/10.1016/j.aam.2009.03.003

29. Qi, F., Guo, B.-N.: Completely monotonic functions involving divided differences of the di- and tri-gamma functions and some applications. Commun. Pure Appl. Anal. 8, 1975-1989 (2009). https://doi.org/10.3934/cpaa.2009.8.1975

30. Qi, F., Guo, B.-N.: Complete monotonicity of divided differences of the di- and tri-gamma functions with applications. Georgian Math. J. 23, 279-291 (2016). https://doi.org/10.1515/gmj-2016-0004

31. Guo, B.-N., Qi, F.: A class of completely monotonic functions involving divided differences of the psi and tri-gamma functions and some applications. J. Korean Math. Soc. 48, 655-667 (2011). https://doi.org/10.4134/JKMS.2011.48.3.655

32. Alzer, H., Grinshpan, A.Z.: Inequalities for the gamma and q-gamma functions. J. Approx. Theory 144, 67-83 (2007). https://doi.org/10.1016/j.jat.2006.04.008

33. Gao, P.: Some completely monotonic functions involving the polygamma functions. J. Inequal. Appl. 2019, 218 (2019). https://doi.org/10.1186/s13660-019-2172-x 
34. Gao, P.: Some monotonicity properties of gamma and q-gamma functions. ISRN Math. Anal. 2011, 375715 (2011). https://doi.org/10.5402/2011/375715

35. Qi, F:: Decreasing properties of two ratios defined by three and four polygamma functions. Comptes Rendus Mathématique Académie des Sciences Paris 359(11) (2021, in press)

36. Elezović, N., Giordano, C., Pečarić, J.: The best bounds in Gautschi's inequality. Math. Inequal. Appl. 3, 239-252 (2000). https://doi.org/10.7153/mia-03-26

37. Liang, L., Zhao, B., Li, A.: Some inequalities involving the polygamma functions. J. Inequal. Appl. 1, 54 (2019). https://doi.org/10.1186/s13660-019-1999-5

38. Qi, F., Agarwal, R.P.: On complete monotonicity for several classes of functions related to ratios of gamma functions. J. Inequal. Appl. 2019, 36 (2019). https://doi.org/10.1186/s13660-019-1976-z

39. Qi, F: Lower bound of sectional curvature of Fisher-Rao manifold of beta distributions and complete monotonicity of functions involving polygamma functions. Results Math. 76(4), 217 (2021). https://doi.org/10.1007/s00025-021-01530-2

40. Trimble, S.Y., Wells, J., Wright, F.T.: Superadditive functions and a statistical application. SIAM J. Math. Anal. 20, 1255-1259 (1989). https://doi.org/10.1137/0520082

41. Guo, B.-N., Qi, F.: Properties and applications of a function involving exponential functions. Commun. Pure Appl. Anal. 8(4), 1231-1249 (2009). https://doi.org/10.3934/cpaa.2009.8.1231

\section{Submit your manuscript to a SpringerOpen ${ }^{0}$ journal and benefit from:}

- Convenient online submission

- Rigorous peer review

- Open access: articles freely available online

- High visibility within the field

- Retaining the copyright to your article

Submit your next manuscript at $\gg$ springeropen.com 